\section{A Minute Rectal Carcinoma with Massive Submucosal Invasion and Lymph-Node Metastasis}

Recently, small and minute colorectal carcinomas, including flat and depressive types, have been detected $(1-3)$, and endoscopic resection methods such as strip biopsy have often been applied to these tumors $(2,4)$. We report here on a rare case of minute rectal carcinoma with massive submucosal invasion and lymph-node metastasis.

A 63-year-old Japanese man was admitted with diarrhea. He underwent a total colonoscopic examination, which identified a sessile polyp, about $5 \mathrm{~mm}$ in size, in the rectum (Figure 1). The polyp appeared to be covered by reddish mucosa, with capillaries, and showed a slight central depression. The clinical diagnosis was mucosal adenocarcinoma, since there was no appearance of ulceration and no massive depression. Strip biopsy was carried out with a submucosal injection of saline beneath the polyp, capturing the artificial elevation including the lesion, surrounding the normal mucosa with a snare, and resection using cutting current.

Histologically, the polyp proved to be a moderately differentiated adenocarcinoma, with massive submucosal invasion (Figure 2). The mucosal carcinoma existed only in the central depression, $3 \mathrm{~mm}$ in size, and the submucosal carcinoma measured $4.5 \mathrm{~mm}$, elevating the mucosal carcinoma and surrounding nonneoplastic mucosa. The resected margin was free of tumor. However, lymphatic spread was found in the superficial submucosa. Since this finding was considered to be a risk factor for lymph-node metastasis, surgery (low anterior resection) was subsequently performed, which revealed a lymph-node metastasis.

The present case shows that, even in minute carcinoma, there is a risk for lymph-node metastases when there is lymphatic vessel invasion in the resected specimen (5). In addition to careful endoscopic observation, precise histological examination is therefore necessary to provide adequate endoscopic treatment for colorectal carcinoma.

\section{K. Yagi', T. Goto', A. Sekine', K. Sakakibara', R. Abe?, Y. Matubara ${ }^{2}$, M. Iwafuchi ${ }^{3}$ \\ 'Dept. of Internal Medicine and \\ ${ }^{2}$ Dept. of Surgery, Prefectural Yoshida Hospital, Yoshidamachi, \\ Nishikanbaragun, Niigata, Japan; \\ Dept, of Biomedical Technology (Pathology), College of \\ Medical Technology of Niigata University, Niigata, Japan}

\section{Corresponding Author}

K. Yagi, M.D., Dept. of Internal Medicine

Prefectural Yoshida Hospital

Yoshidamachi

Nishikanbaragun, Niigata-ken 959-02

Japan

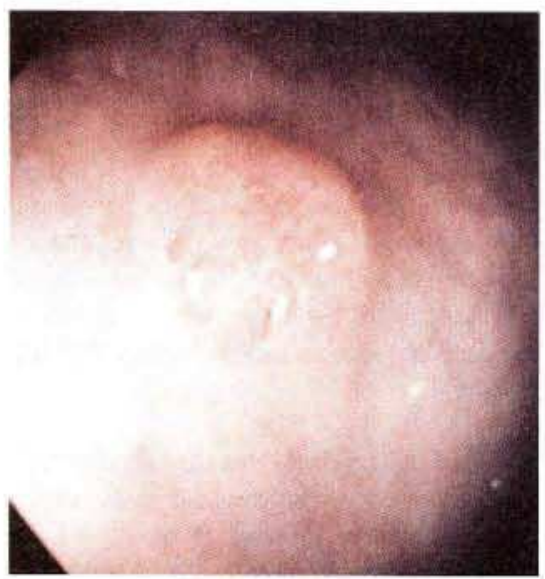

Figure 1: An endoscopic view, showing a sessile polyp with a central depression.

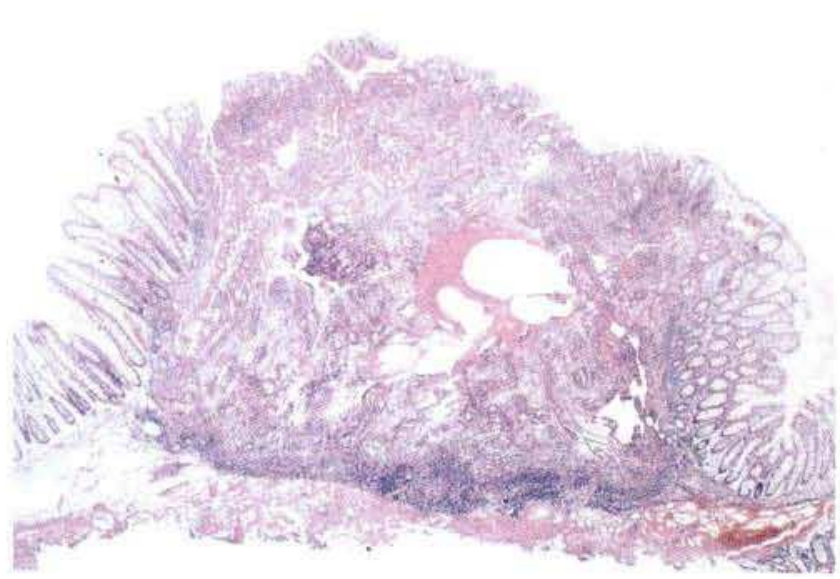

Figure 2: Histological section of the resected specimen. The photomicrograph shows adenocarcinoma extending into the submucosa, with the mucosal carcinoma in the depressed part (hematoxylin-eosin, $x$ 10)

\section{References}

1. Shimoda T, Ikegami M, Fujisaki J, et al. Early colorectal carcinoma, with special reference to its development de novo. Cancer 1989; 64: 1138-46.

2. Kudo S. Endoscopic mucosal resection of flat and depressed types of early colorectal cancer. Endoscopy 1993; 25: 455-61.

3. Karita M, Tada M, Okita K, et al. Endoscopic therapy for early colon cancer: the strip biopsy resection technique. Gastrointest Endosc 1991; 37: 128-32.

4. Minamoto T, Sawaguchi K, Ohta T, et al. Superficial-type adenomas and adenocarcinomas of the colon and rectum: a comparative morphological study. Gastroenterology 1994; 106 : $1436-43$.

5. Sugihara K, Muto T, Morioka Y, et al. Management of patients with invasive carcinoma removed by colonoscopic polypectomy. Dis Colon Rectum 1989; 32: 829-34. 